\title{
O PAPEL DA TRADUÇÃO NO ENSINO DE LÍNGUAS ESTRANGEIRAS MODERNAS
}

\author{
EL PAPEL DE LA TRADUCCIÓN EN LA ENSEÑANZA DE LENGUAS \\ EXTRANJERAS MODERNAS
}

\author{
THE ROLE OF TRANSLATION IN TEACHING MODERN FOREIGN \\ LANGUAGES
}

Glauber LIMA MOREIRA ${ }^{1}$

RESUMO: Esta entrevista com a professora Dra. Janet DeCesaris, pesquisadora do Departamento em Tradução e Ciências da Linguagem da Universitat Pompeu Fabra (UPF) e atualmente diretora do Institut Universitari de Lingüística Aplicada (IULA), tem como objetivo apresentar o seu contato com a tradução e a sua concepção sobre o uso da tradução no ensino de línguas estrangeiras modernas no contexto atual.

PALAVRAS-CHAVE: Tradução. Experiência profissional. Língua estrangeira. Ensino de línguas.

RESUMEN: Esta entrevista con la profesora Dra. Janet DeCesaris, investigadora del Departamento en Traducción y Ciencias del Lenguaje de la Universitat Pompeu Fabra (UPF) y actualmente directora del Institut Universitari de Lingüistica Aplicada (IULA), tiene como objetivo presentar su contacto con la traducción y su concepción sobre en el uso de la traducción en la enseñanza de las lenguas extranjeras modernas en el contexto actual.

PALABRAS CLAVE: Traducción. Experiencia professional. Lengua extranjera. Enseñanza de lenguas.

ABSTRACT: This interview with Professor Dr. Janet DeCesaris, researcher of the department in Translation and Language Sciences from the Universitat Pompeu Fabra $(U P F)$ and she is currently the principal of the Institut Universitari de Lingüistica Aplicada (IULA), aims to present her contact with translation and her conception about the use of translation in the teaching of modern foreign languages in the current context.

1 Universidade Federal do Piauí - (UFPI), Campus Ministro Reis Velloso, Parnaíba - PI - Brasil. Profesor de Lengua Española en Curso de Bacharelado em Turismo. Doctorando en Traducción y Ciencias del Lenguaje en la Universitat Pompeu Fabra (UPF) con beca CAPES. E-mail: glauberlimamoreira@gmail.com. 
KEYWORDS: Translation. Professional Experience. Foreign Language. Language teaching.

\section{Introducción}

Presentamos esta entrevista con la Dra. Janet DeCesaris, profesora del programa de doctorado en Traducción y Ciencias del Lenguaje y actualmente directora del IULA. Institut de Lingüística Aplicada - Centro Específico de Investigación de la Universitat Pompeu Fabra (UPF). Es miembro del Grupo de Investigación Infolex (Grup de Recerca en Informació Lexicogràfica) de la misma institución superior. Sus líneas de investigación son: Lexicografía, morfología, y aplicación de la linguiística teórica a la traducción. La temática es acerca de la traducción y tiene como objetivo presentar el contacto de la entrevistada con la traducción y su concepción sobre en el uso de la traducción en la enseñanza de las lenguas extranjeras modernas en el contexto actual.

Glauber Lima Moreira: ¿Cómo fue su experiencia con la práctica de la traducción?

Janet DeCesaris: Ejercí como traductora profesional, principalmente del español y del catalán al inglés, durante varios años. Trabajé con varias agencias de traducción en Barcelona y alrededores y la mayoría de los textos eran del campo empresarial, o bien cartas comerciales o bien descripciones de productos para la exportación. Durante muchos años transcribí i traduje del inglés al español películas pedagógicas para la enseñanza de técnicas de enfermería. Adapté y traduje el libro de Terminología de $M^{a}$ Teresa Cabré y varias obras de teatro escritas originalmente en catalán. En conjunto, como muchos otros traductores, he trabajado con textos de varios campos temáticos. La traducción siempre me ha interesado porque obliga a considerar la misma realidad desde más de una perspectiva.

Glauber Lima Moreira: ¿Qué concepción tiene sobre traducción?

Janet DeCesaris: Creo que la traducción consiste en intentar reproducir una idea expresada en una lengua determinada en otra. Se puede conseguir una traducción muy fidel en textos que no tienen un alto grado de contenidos culturales, pero en la mayoría 
de textos, la suma del componente cultural y la idiosincrasia de cada lengua hacen que la traducción sea una actividad muy difícil. Casi siempre se pierde alguna cosa en la traducción, pero los buenos traductores saben compensar esta pérdida casi sistémica.

Glauber Lima Moreira: Desde hace un tiempo la traducción ha sido marginalizada en la enseñanza de lenguas extranjeras modernas (LEM). ¿Le parece interesante defender su uso en la enseñanza de LEM en la actualidad?

Janet DeCesaris: Evidentemente. Hoy en día se prioriza la aproximación comunicativa, que es apropiada para mucha gente. Pero casi se ha llegado a abandonar por completo la traducción como actividad formativa dentro de un programa general de aprendizaje de lenguas extranjeras. No solo es una lástima, es una laguna en la formación porque con la tecnología moderna, que ha hecho posible la transmisión casi inmediata de la palabra escrita, lo escrito vuelve a ocupar un sitio imprescindible en casi todos los campos. En la actualidad los empleadores tienen la expectativa que los trabajadores con lenguas tengan tener cierta práctica con la traducción porque muchos empleos dependen, casi exclusivamente, de la palabra escrita.

Glauber Lima Moreira: ¿Cómo y cuándo es conveniente abordar el tema de la traducción en la enseñanza de una lengua extranjera?

Janet DeCesaris: A mí me parece que es conveniente abordarlo a niveles avanzados, como mecanismo para comparar lenguas y para concienciar a los alumnos de la complejidad de la expresión lingüística. Se puede introducir como parte de unidades didácticas de comparación. Hay que introducir la traducción primero con la comparación de oraciones para luego comparar textos cortos. Es interesante trabajar primero con la comparación de textos traducidos, para que los alumnos puedan ver la complejidad y los factores que hay que tener en cuenta. Un error frecuente en la clase de lenguas extranjeras es dar tareas de traducción sin haber preparado al alumnado, sin haberle proporcionado las herramientas apropiadas para traducir. Sin la 
preparación adecuada, esos ejercicios casi siempre son un fracaso pedagógico y solo sirven para aumentar el nivel de frustración del alumnado.

Glauber Lima Moreira: ¿Qué resultados positivos se pueden obtener con la aplicación de la traducción en el aula?

Janet DeCesaris: Un alumnado concienciado de las diferencias entre lenguas siempre es positivo. Y diría que es imposible llegar a tener esa conciencia sin haber trabajado con la traducción.

Una de las grandes ventajas de trabajar con la traducción en el aula es que se ve muy claramente las diferencias entre el léxico de una lengua y de otra. La equivalencia léxica entre lenguas es algo que suele existir solo en el vocabulario de campos de especialidad y es muy poco frecuente, para no decir inexistente, en el léxico común. Le doy un ejemplo. La palabra "verde" en español tiene varias acepciones y es frecuente en la fraseología, por ejemplo, en la frase "chiste verde”. En inglés para referirse al mismo tipo de chiste no se utiliza la voz “green”. ¿Qué quiere decir esto? ¿Qué "green" no es el equivalente de "verde"? En efecto, al hablar de chistes "green” no es el equivalente de "verde" pero sí lo es al referirse al color del césped. Al traducir se ven muchos ejemplos parecidos, lo cual hace que el alumno tenga más conciencia de la expresión genuina en cada idioma.

Glauber Lima Moreira: ¿La traducción puede ofrecer actividades que favorecen efectivamente al aprendizaje del estudiante referente a las cuatro destrezas lingüísticas?

Janet DeCesaris: La traducción es más apropiada para la lengua escrita que para la lengua oral. En cuanto a la lengua escrita, hay que distinguir entre traducción directa, es decir, la traducción hacia la lengua materna, y traducción inversa, que se hace hacia una lengua extranjera. Ambas direcciones tienen aplicaciones didácticas interesantes, pero son diferentes. Hay que tener en cuenta que la traducción de oraciones sueltas puede focalizar el interés de los alumnos en una diferencia importante entre lenguas, pero en general me gusta mucho más plantear cuestiones de traducción en un contexto 
pedagógico con un nivel de texto más amplio, como ahora el párrafo. La traducción como actividad de aprendizaje tiene más que ver con la comprensión lectora y la producción escrita, que con la comprensión y producción orales. Por eso se llaman intérpretes y no traductores los que trabajan en la transmisión oral.

Glauber Lima Moreira: ¿Cuáles son los desafíos de los profesores de LE de trabajar la traducción en clase?

Janet DeCesaris: Creo que el mayor desafio consiste en saber proporcionar suficiente contexto como para el alumno pueda hacer una traducción buena. A menudo se trabaja con la traducción de frases sueltas, lo cual casi siempre es un error porque una frase nunca existe fuera de un contexto más amplio.

Glauber Lima Moreira: ¿La traducción debe ser presentada, en la enseñanza de lenguas, cómo una herramienta didáctica o como la quinta destreza integrada a las cuatro destrezas lingüísticas clásicas?

Janet DeCesaris: Me inclino por trabajar la traducción en un contexto pedagógico como una herramienta de aprendizaje. La enseñanza de la traducción en un contexto más profesionalizador es otra cosa y para muchos que quieren desarrollar su competencia en lengua extranjera, no es necesario poder traducir con criterios profesionales. La tipología de textos en la traducción profesional no coincide con los intereses de muchos alumnos de lengua extranjera, y así creo que es mejor considerar la traducción en el contexto de enseñanza de lenguas extranjeras como una herramienta y no como un fin en si mismo.

Glauber Lima Moreira: En su práctica docente, ¿cómo haría para inserir la traducción para las clases de lenguas extranjeras?

Janet DeCesaris: Como ejercicios de análisis y práctica, a partir del tercer año de estudio universitario o bien a un nivel avanzado que, según cómo, puede ser anterior al 
tercer año. Es preferible trabajar con temas que ya son conocidos entre los alumnos, como los que salen en las noticias, en que se utiliza el léxico común y no especializado. Hay que empezar con temas conocidos en una primera etapa para reducir la complejidad de la traducción en un contexto de aprendizaje de lenguas.

Glauber Lima Moreira: ¿Puede contarnos un poco sobre la creación del programa de Traducción y Ciencias del Lenguaje en la Universitat Pompeu Fabra?

Janet DeCesaris: Se creó la Facultad de Traducción e Interpretación de la UPF hace más de 20 años, con el encargo de impartir la licenciatura oficial en Traducción e Interpretación. Con la implementación del Plan Bolonia en Europa, ahora impartimos el grado oficial en Traducción e Interpretación, y también otro grado en Lenguas Aplicadas. Los programas de posgrado son más especializados, y tenemos un Máster en Traducción, otro en Lingüística Teórica y Aplicada, otro en Estudios del Discurso y también otro, impartido conjuntamente con la Universidad de Barcelona, en la enseñanza del español como lengua extranjera. La UPF decidió crear una Facultad de Traducción e Interpretación para dar respuesta a la necesidad creciente en Cataluña de tener profesionales con un perfil multilingüe.

Glauber Lima Moreira: ¿El programa de doctorado en Traducción y Ciencias del Lenguaje de la UPF discute/trata sobre la utilización de la traducción en las clases de LE? ¿Podría citar algunas de las propuestas y/o proyecto de investigación que actualmente están en desarrollo en la universidad?

Janet DeCesaris: Que yo sepa, la mayoría de doctorandos en nuestro programa no trabajan ese tema en sus tesis. En general, las tesis o son de Lingüística teórica o aplicada, o bien son estudios de estrategias de traducción empleadas en un tipo de obra, pero sorprendentemente no hay mucha gente interesada en el estudio de la traducción como herramienta pedagógica en la enseñanza de lenguas extranjeras. 


\section{Consideraciones finales}

La presente entrevista con la profesora e investigadora Dra. Janet DeCesaris señala la importancia de la utilización de la traducción en el contexto escolar como herramienta pedagógica de apoyo al profesor de lenguas extranjeras para que pueda desarrollar una clase más eficiente y motivadora, y também para que el estudiante tenga más un instrumento de aprendizaje.

Durante toda la discusión en el texto vemos que la traducción es considerada por la entrevistada como más un recurso didáctico que funciona como mecanismo de referencia para la enseñanza de las destrezas linguísticas de los aprendices.

Por tanto, para la professora investigada la traducción debe ser considerada como actividad de aprendizaje que tiene más que ver con la comprensión lectora y la producción escrita, que con la comprensión y producción orales, y que es mejor considerar la traducción en el contexto de enseñanza de lenguas extranjeras como una herramienta y no como un fin en si mismo.

\section{REFERENCIAS}

CABRÉ, M. T. La Terminología: teoría, metodología, aplicaciones. Barcelona: Editorial Antártida/Empúries.

\section{Como referenciar este artigo}

LIMA MOREIRA, Glauber. O papel da tradução no ensino de línguas estrangeiras modernas. Rev. EntreLínguas, Araraquara, v. 3, n. 2, p. 352-358, jul./dez. 2017. Disponível em: <https://doi.org/10.29051/rel.v3.n2.2017.9109>. E-ISSN: 2447-3529.

Submetido em: 16/01/2017

Revisões requeridas: 13/03/2017

Aprovado em: 12/04/2017 